\title{
The theory of thermodynamic and magnetic properties of multi-band superconductors
}

\author{
M.E. Palistrant* \\ Institute of Applied Physics, Str. Academiei 5, Chishinau MD 2028, Republic of Moldova
}

Received September 10, 2009, in final form November 2, 2009

The main stages of development of the theory of superconducting systems with overlapping energy bands are formulated. The main references of the classical papers of the authors of this theory (Prof. V.A.Moskalenko and his co-workers) are listed.The list also includes papers related to high-temperature superconductivity. Some peculiarities of the two-band model, which give qualitatively new results in comparison with the usual oneband model, are described.The application of the two-band model for the description of the thermodynamical and magnetical properties of the compound $\mathrm{MgB}_{2}$ is also discussed.

Key words: multi-band superconductivity, thermodynamic properties, low carrier densities, upper critical field, $M g B_{2}$

PACS: $74.25 . \mathrm{Ha}, 74.20 . \mathrm{Fg}, 74.70 . \mathrm{A}$

\section{Introduction}

The mechanism of such remarkable effect as superconductivity was discovered (in 1957). This problem was solved as a result of the long-time and intense theoretical and experimental researches (it took almost 50 years) and it appeared as the most important cryogenics discovery. A great merit in the construction of the theory of superconductivity belongs to the great physicist and mathematician N.N. Bogolyubov [1-4], whose centenary we celebrate nowadays. Also, great advances in the development of the theory belong to his disciples: S.V. Tiablikov, D.N. Zubarev, D.V. Shiarkov and other eminent scientists. Along with the development of the theory of superconductivity in the isotropic systems, Bogolyubov came up with the idea of a necessity to take into account the anisotropic properties of superconductive systems, which made it possible to describe the real metals. The first work in this direction belongs to V. Moskalenko, where the anisotropy appears in the band structure of the considered system.

The model of a superconductor with overlapping energy bands on the Fermi surface was proposed by about 50 years ago V. Moskalenko [5] and after a while also by H. Suhl et al. [6] .

This model considers the anisotropy of energy bands and describes the superconducting properties of transitional metals that have two or more groups of electrons belonging to different energy bands on the Fermi surface.

The main assumption of the model is the formation of Cooper pairs of electrons inside one energy band and transition of this pair as a whole entity from one energy band to another. This results in the appearance of the intraband $V_{n n}$ and the interband $V_{n m}(m \neq n, n ; m=1,2)$ electronic interactions that lead to the additional attraction of electrons which, in its turn, favors an increase of the superconducting transition temperature. This is equivalent to the diagonal approximation over band indices in the base equations of the theory; two order parameters $\Delta_{11}$ and $\Delta_{22}$ appear in the two-band model.

Having made these assumptions, Moskalenko and his co-workers carried out investigations of the thermodynamic and electromagnetic properties of the many-band superconductors. A few books and a lot of articles on this problem have been published (see, for example, [7-16] and references therein as well as in $[17,18])$.

\footnotetext{
*E-mail: mepalistrant@yandex.ru
} 
A new scientific trend in the studies of multiband superconductor properties appeared. Along with Moldavian theoretical physicists, there were a lot of scientists in different countries who worked in this direction (see for example [19-22] and references in book review [15]). It should be noted that the most valuable contribution to the development of the theory of superconductivity in the systems where two and more energetic bands overlap on the Fermi surface was made by Moldavian scientists.

\section{The two-band high-temperature superconductivity}

At the time preceding the discovery of high- $T_{\mathrm{c}}$ superconductivity, the theory of superconductivity with overlapping energy bands was developed in order to describe the physical characteristics in the systems with heavy fermions [23,24]. The discovery of high- $T_{\mathrm{c}}$ superconductivity [25] has compelled attention to this problem again. The rising tide of interest is explained by the overlapping of two or more energy bands on the Fermi surface in the high- $T_{\mathrm{c}}$ superconductor materials. Such a conclusion results from band structure of oxide ceramics [26,27]. This can be understood as well, if we consider the layered structure of these systems.

The Hamiltonian that describes the system with $n$ layers and takes into account interactions of electrons inside each layer and between layers can be easily transformed into the Hamiltonian that describes $n$ band system [28-30]. This transformation was done by the diagonalization of the Hamiltonian of a layer system and the introduction of the symmetrical and anti-symmetrical states of electrons.

Since the discovery of high- $T_{\mathrm{c}}$ superconductivity, a great number of theoretical studies of manyband model [5] regarding the high- $T_{\mathrm{c}}$ materials have been carried out.

Series of experimental studies have been performed as well. These studies revealed the presence of two energy gaps in the energy spectrum of electrons and proved, in turn, the appearance of singularities caused by the overlapping of energy bands on the Fermi surface in the energy spectrum of electrons (see, for example, [31]).

Nowadays, researchers have concluded that an experiment in oxide metals does not confirm the presence of two energy gaps. It may be the result of many difficulties of the high- $T_{\mathrm{c}}$ superconductivity and, in particular, of the disorder in the system (great concentrations of impurity or oxygen deficiency). In this case, despite the presence of two order parameters, the only one energy gap is found in two-band superconductors at high concentrations of impurity [13]. The averaged order parameter $\bar{\Delta}=\frac{N_{1} \Delta_{1}+N_{2} \Delta_{2}}{N_{1}+N_{2}}$ that determines the averaged energy at $T=0$ [32] appeared.

$$
\frac{E_{\mathrm{S}}-E_{\mathrm{N}}}{V}=-\frac{1}{2} \bar{\Delta}^{2}\left(N_{1}+N_{2}\right),
$$

where $N_{i}$ is the density of electron states on the $i$ th fold of the Fermi surface.

At great concentrations of impurity the jump of heat capacity at the critical point $T=T_{\mathrm{c}}$ in the two-band system has the following form [13]

$$
\frac{C_{\mathrm{S}}-C_{\mathrm{N}}}{C_{\mathrm{N}}}=\frac{8 \pi^{2}}{7 \beta_{\mathrm{c}}} \frac{N_{1}+N_{2}}{\zeta(3)} .
$$

These expressions formally coincide with the case of the pure one-band model with the only difference that quantity $\beta_{\mathrm{c}}=\left(k T_{\mathrm{c}}\right)^{-1}$ is determined against the two-band background and contains all electron-electron interaction couplings (the intraband and interband ones). In addition, the density of electron states is replaced by the total density of both bands. Note that in the two-band systems with great concentrations of non-magnetic impurity the equation of Ginsburg-Landau is reduced to the one-band equation for the wave function $\Delta(\mathbf{x})$ that is the gravity center of wave functions $\Delta_{1}(\mathbf{x})$ and $\Delta_{2}(\mathbf{x})$. In this way, we have to take into account that the temperature $T_{\mathrm{c}}$ and the parameter $\kappa$ of this one-band equation are determined on the two-band basis.

Therefore, the overlapping of energy bands on the Fermi surface significantly contributes to the physical quantities even at strong disorder in the system. 
The conclusion on the absence of two gaps in the electron energy spectrum or even the exclusion of the contribution of both energy bands that overlap on the Fermi surface to the thermodynamic and electromagnetic characteristics of the oxide metals has been stated earlier. More detailed experimental studies should be done both at high and low densities of charge carriers in order to change the disorder of a two-band system.

Note also that Moskalenko's model [5], as well as its generalizations for the arbitrary densities of charge carriers (including very low densities), assumes the singlet pairing in Cooper pairs. There is no difficulty to build the theory of the two-band model with $d$ pairing of electrons as it has been done for the two-band superconductors with non-magnetic impurity [33].

The consideration of the overlapping of the energy bands leads not only to the quantitative difference of the results from the case of the one-band superconductor but, in some cases, to qualitatively new results. For example:

1. In two-band superconductors, the high temperatures of the superconducting transition are possible not only in the case of attractive interaction between electrons, but even in the case of repulsive one $\left(\lambda_{n m}<0, n, m=1-2\right)$, when the relationship $\lambda_{11} \lambda_{22}-\lambda_{12} \lambda_{21}<0$ is fulfilled $[13,42]$.

2. In impurity two-band superconductors, for example, the Anderson theorem is violated at $\Delta_{1} \neq \Delta_{2}$, and the dependence of the thermodynamic quantities on concentration of the non-magnetic impurity due to the interband scattering of electrons on the impurity atoms is observed.

3. In two-band superconductors, collective oscillations of the exciton-like Leggett mode [74] caused by fluctuations of the phase of order parameters from different bands are observed. In three-band systems, as well as in the two-band case with due regard for the Cooper pairs of electrons from different energy bands, which is reduced to the effective three-band model, the number of such oscillatory modes can be two [81-85].

4. Using the two-band model and assuming the moderate values of the coupling constants, one can obtain high values of $T_{\mathrm{c}}$, the two energy gaps $2 \Delta_{1} / T_{\mathrm{c}}>3.5$ and $2 \Delta_{2} / T_{\mathrm{c}}<3.5$, large negative values $\mathrm{d} \ln T_{\mathrm{c}} / \mathrm{d} \ln V$ ( $V$ is the volume), positive curvature of the upper critical field near the transition temperature, etc. [34-38]. Furthermore, in the two-band model, it is possible to describe the decrease of $T_{\mathrm{c}}$ with increasing system disorder [39-41].

5. The location of the Fermi level that can be changed by doping plays an important role in determining the thermodynamic and magnetic properties of the two-band superconductor. Having assumed a non-phonon pairing mechanism of superconductivity, as well as a phonon mechanism in the many-band systems with the lowered densities of the charge carriers, the account of the singularities mentioned above is very crucial. A particular interest is attached to the possibility to obtain a bell-shaped dependence of $T_{\mathrm{c}}$ and of the jump of heat capacity $\left(C_{\mathrm{S}}-C_{\mathrm{N}}\right) / C_{\mathrm{N}}$ at the point $T=T_{\mathrm{c}}$ on the carrier density $[41,42,44]$. In the three-band model, it is possible to obtain a "step" in the dependence of $T_{\mathrm{c}}$ on the carrier density $[45,46]$. The investigation of the properties of superconductors with overlapping energy bands on the Fermi surface and the electronic topological transitions was reviewed in [47]. This review contains the classical results of the problem.

An increase in the number of energy bands on the Fermi surface increases the overall density of electron states and leads to the onset of an additional interband electron-electron interaction that contributes to the onset of superconductivity. This interaction violates the universal BCS relations and leads to the substantial dependence of a number of physical characteristics on properties of the anisotropic system $[12,13,15,47-49]$.

The discovery of superconductivity in the intermetallic borocarbide nitrides was the important whirling point in superconductivity. These compounds possess interesting superconducting and magnetic properties. A more detailed information on the discovery of new diverse intermetallic compounds and their properties can be found in book [51]. 


\section{Superconductivity in the compound $\mathrm{MgB}_{2}$}

The discovery of superconductivity in the intermetallic compound $\mathrm{MgB}_{2}$ was even more intriguing [52]. The main physical properties and the singularities are as follows (see for example, [53] and [54]):

1. High temperatures of the superconducting transition $T_{\mathrm{c}} \sim 39 \mathrm{~K}[52]$.

2. The interaction responsible for the formation of superconducting pairs is caused by an exchange of phonons [55,56], and the symmetry of the Cooper pairs is of the $s$-wave type [57].

3. The average phonon frequencies are 2-3 times as high as for classical superconductors $\mathrm{Nb}_{2} \mathrm{Sn}$ $[58,59]$, the mass renormalization factor $(1+\lambda)$ is small, the Sommerfeld's coefficient $\gamma_{n}$ and the condensation energy also appear to be inconsistent with superconductivity near $\sim 40 \mathrm{~K}$.

Researchers have concluded that the superconductivity in $\mathrm{MgB}_{2}$ cannot be understood from the isotropic one-band BCS model. The anisotropy of the system is the key factor here that is manifested in the anisotropy of energy bands (the overlapping of energy bands on the Fermi surface) and in the anisotropy of the order parameter, i. e., its dependence on the momentum direction.

As stated above, the properties of isotropic two-band (multi-band) superconductors had been studied long before the discovery of $\mathrm{MgB}_{2}$ compounds by Moskalenko and his co-workers. These studies show that the thermodynamic and electromagnetic properties of the two-band superconductor are qualitatively different from those of the one-band superconductor.

Another field of studies in BCS theory is an account of anisotropy of electron- phonon interaction $[60,66]$. The band anisotropy, as well as the anisotropy of matrix element of electron-phonon interaction, is shown in the works mentioned above to decrease the relative jump of electron heat capacity $\left(C_{\mathrm{S}}-C_{\mathrm{N}}\right) / C_{\mathrm{N}}$ at $T=T_{\mathrm{c}}$ in comparison with the value of 1.43 , which is specific for the isotropic case.

The method of calculating electron heat capacity that permits to take into consideration the existence of greater number of bands and the anisotropy of energy gaps in each band has been developed in works [62-64].

We did not aim to make a thorough analysis of the works mentioned above. Note only that a clear picture with regard to the band structure, the topology of the Fermi surface, the values of densities of electron states, the averaged velocities of electrons on the Fermi surface, and other characteristics necessary for consistency of theory with experiment has been obtained. $\operatorname{In} \mathrm{MgB}_{2}$ two energy gaps $\Delta_{1}(0)=6,8 \mathrm{meV}\left(2 \Delta(0) / k_{\mathrm{B}} T_{\mathrm{c}}=4,0\right) ; \Delta_{2}(0)=1,8 \mathrm{meV}\left(2 \Delta_{2}(0) / k_{\mathrm{B}} T_{\mathrm{c}}=1,06\right)$ have been experimentally confirmed [65]. As a result, some singularities of thermodynamic properties have been observed in these compounds. The shoulder-type anomaly, for example, appears in the temperature dependence of the heat capacity around $0.25 T_{\mathrm{c}}$ as well as $\left.\left(C_{\mathrm{S}}-C_{\mathrm{N}}\right) / C_{\mathrm{N}}\right) \approx 0,8$ at $T=T_{\mathrm{c}}$ [59], which is consistent with the theory [5,9,64]. Another experimentally observed anomaly is the positive curvature of the upper critical magnetic field $H_{c_{2}}(T)$ near the temperature of superconducting transition (see theoretical studies $[47,65]$ ). Note also the breakdown of the Anderson theorem in the two-band system with the non-magnetic impurity due to the interband scattering of electrons on impurity that leads to the disorder in the system $[7,8,13]$. These and other anomalies in $\mathrm{MgB}_{2}$ can be understood only considering the overlapping of energy bands on the Fermi surface. Two-band model qualitatively describes the main singularities in the behavior of the physical characteristics of $\mathrm{MgB}_{2}$.

A possibility to describe superconductivity on the basis of two-band model in other compounds cannot be excluded. This fact validates the following generalizations and the development of superconductivity theory taking into account the overlapping of energy bands.

\section{Properties of superconductors with reduced density of charge carriers}

In all the above mentioned works, the two-band model was used to describe the properties of the superconductors for which the relationship $\mu \gg T_{\mathrm{c}}$ is satisfied ( $\mu$ is the chemical potential). 
This description is made in the diagonal approximation over the band indices $[8,13]$.

In systems with low carrier density, however, the relation $\mu \gg T_{\mathrm{c}}$ does not hold. Therefore, to develop the theory of superconductivity for the two-band systems without restrictions on the Fermi energy, we consider simultaneously two possible superconductivity mechanisms - phonon and electron ones. A substantial dependence of the chemical potential $\mu$ on the order parameter in the superconducting phase is an inherent feature of the systems with low carrier density. These circumstances have been noted in many papers, and the feasibility of experimental observation of these anomalies in the temperature dependence of the chemical potential was first suggested in [66]. By the example of the BCS model, it was shown there that the $\mu(T)$ curve has an experimentally observable bend at the point $T=T_{\mathrm{c}}$. Below we shall show that this effect in the two-band case is enhanced by the presence of two or four order parameters $\left(\Delta_{n m} ; m=1,2\right)$ and is manifested at the $\mu$ values more easily observed in experiment [67].

In the articles cited above, investigations were carried out in the scenario of Cooper pairing. In the systems with small carrier concentrations, the bound states may arise following the decrease in the carrier concentration, and the transition to Bose condensate of localized pairs with the finite bound energy may occur (the Schaffroth scenario [68]). The possibility of such a transition in the one-band systems was discussed in a number of articles (for example ([67-74]).

As is shown in [69] and [72], the change of sign in the chemical potential with the decrease in the carrier concentration corresponds to the transition from the BCS to the Schaffroth scenario. Condensation of localized pairs occurs at the concentrations of carriers for which $\mu \leqslant 0$.

We present the theory of superconductivity of a two-band system that is valid at any carrier density and takes into account all possible pairing of electrons due to the intraband and the interband interactions within the Cooper pairing scenario. The critical temperature $T_{\mathrm{c}}$, the chemical potential $\mu$, the heat capacity $\left(C_{\mathrm{S}}-C_{\mathrm{N}}\right)$ at the point $T=T_{\mathrm{c}}$ as a functions of the carrier density are shown $[47,67,75]$. This theory is used to describe the properties of the compounds $\mathrm{MgB}_{2}$ where magnesium and boron are replaced by different elements of the periodic table. The articles [76] and [77] are designed for the self-consistent discussion in the mean-field approximation of the system of equations for the order parameters $\Delta_{n}$ and $\mu$ at $T=0$. There is revealed the effect of the overlapping of energy bands on these quantities and the carrier concentration at which the system experiences the transition from the Cooper pairing $(\mu>0)$ to the Schaffroth $(\mu<0)$ scenario. The equation for the binding energy $\varepsilon_{b}$ of the two-particle state is also obtained, and the relationship between $\varepsilon_{b}$ and $\mu$ is established.

The path integral method as applied to the two-band model is also developed; on this basis, the procedure for the transition from the Fermi to Bose elementary excitations at $T=0$ is given $[75,78,79]$. The Bose system condensation temperature $T_{k}$ is also determined. The theory of superconductivity in two-band non-adiabatic systems with strong electron correlations in the linear approximation over non-adiabaticity is built [80].

Along with the above described phenomena related to the energy bands overlapping on the Fermi surface, we must examine a very interesting thing - the appearance of collective oscillations due to the phase fluctuations of the order parameters of different bands. Firstly this phenomenon was studied in the theoretical work by Leggett [74]. According to its nature, it can appear only in the systems with two or more energy bands on the Fermi surface. Our following results on collective oscillations in three-dimensional systems and in the systems with reduced dimensionality develop the Leggett's studies considering two or more energy bands, at phonon and non-phonon superconducting mechanisms, supposing a reduced and a weak carrier concentration up to the transition from BCS state $(\mu>0)$ to the Shafrott state $(\mu<0)$. The collective exciton-type modes differ quantitatively in different systems and are determined by physical features of the examined systems [81-85]. 


\section{Thermodynamic and magnetic properties of the superconducting doped $\mathrm{MgB}_{2}$ compound}

It should be noted that the above discussed two-band model [5] was found to be very useful; it explained a lot of abnormal physical properties of superconductive anisotropic systems and gave a quite good accordance with experimental data.

Let us present examples of the description of thermodynamic and magnetic properties of $\mathrm{MgB}_{2}$, when $\mathrm{Mg}$ and B are replaced by other chemical elements.

\section{a) Thermodynamic Properties of the doped $\mathrm{MgB}_{2}$ compound}

The theory of two-band superconductors with variable or small density of charge carriers [28, $33,42]$ can describe the behavior of thermodynamic quantities, such as $T_{\mathrm{c}}, \Delta_{1}, \Delta_{2},\left(C_{\mathrm{S}}-C_{\mathrm{N}}\right) / C_{\mathrm{N}}$ at $T=T_{\mathrm{c}}$ as functions of the chemical potential or the charge carrier density in $\mathrm{MgB} 2$.

To this end, it is necessary to do the following:

1. Start with the system of equations for the BCS-type order parameters $\Delta_{n}(\mathrm{n}=1,2)$ for the two-band model with the electron-phonon interaction constants $\lambda_{n m}$ corresponding to the strong electron-phonon coupling renormalized in the two band case and to the presence of Coulomb interaction $\mu_{n m}^{*}[86,87]$.

2. Add an equation that determines the chemical potential to the system of equations for the quantities $\Delta_{n}$. This addition is necessary for the systems with the low charge carrier density $\mu \sim \Delta_{n}$. The compound $\mathrm{MgB}_{2}$ is not such a system, because for a pure substance $\mu=\mu_{0} \approx$ $0,74 \mathrm{eV}$; that is, $\mu \gg \Delta_{n}$. However, the additional equation would be introduced by reason of the specific band structure of the considered system: the upper boundary of the $\sigma$-band, which is responsible for superconductivity in $\mathrm{MgB}_{2}$, is located in the vicinity of $\mu_{0}$. This circumstance plays a decisive role in the dependence of the thermodynamic quantities on the parameter $\mu$ varying at substitution of magnesium and boron atoms by chemical elements of another valence.

3. Consider the overlapping of the two-dimensional $\sigma$ and three-dimensional $\pi$-bands on the Fermi surface. The dependence of the thermodynamic quantities on the chemical potential $\mu$ should be derived taking into consideration its proximity to $\mu_{0} \approx 0.74 \mathrm{eV}$ for pure $\mathrm{MgB}_{2}$. From the experimental data for the renormalized constant of the electron-phonon interaction, we obtain $\lambda_{11}=0.302, \lambda_{22}=0.135, \lambda_{12}=0.04, \lambda_{21}=0.038$.

4. Introduce the relative charge carrier density $\delta=\left(\mu-\mu_{0}\right) / \mu_{0}$ that coincides with the corresponding value, which is calculated taking into account the valence of the elements constituting the compounds $\mathrm{Mg}_{1-x} \mathrm{Li}_{x} \mathrm{~B}_{2}, \mathrm{Mg}_{1-x} \mathrm{Cu}_{x} \mathrm{~B}_{2}, \mathrm{Mg}_{0.8} \mathrm{Li}_{0.2} \mathrm{~B}_{2-x} \mathrm{C}_{x}, \mathrm{Mg}_{0.95} \mathrm{Cu}_{0.05} \mathrm{~B}_{2-x} \mathrm{C}_{x}$, and $\mathrm{MgB}_{2-x} \mathrm{C}_{x}$ at different values of $x$. The dependences of the $T_{\mathrm{c}}$ values on $\delta$ built in this way allow us to compare our theoretical results with the experimental data (see, e. g., [88]). As follows from our calculations, doping of $\mathrm{MgB}_{2}$ with electrons $(\delta>0)$ leads to a decrease of the critical temperature $T_{\mathrm{c}}\left(\mathrm{MgB}_{2-x} \mathrm{C}_{x}\right.$ and $\left.\mathrm{Mg}_{0.95} \mathrm{Cu}_{0.05} \mathrm{~B}_{2-x} \mathrm{C}_{x}\right)$, but at doping with holes $(\delta<0)$ the value of $T_{\mathrm{c}}\left(\mathrm{Mg}_{1-x} \mathrm{Li}_{x} \mathrm{~B}_{2}\right)$ does not vary with the parameter $\delta$. This scheme does not cover the compound $\mathrm{Mg}_{0.8} \mathrm{Li}_{0.2} \mathrm{~B}_{2-x} \mathrm{C}_{x}$, in which $T_{\mathrm{c}}$ at $\delta=-0.02$ takes the value 39.4 (that corresponds to $\mathrm{MgB}_{2}$ ) and decreases with the hole density reduction.

Our theory takes into account the factor of the energy band occupation (variation of the chemical potential $\mu$ ) and scattering of the charge carriers on the impurity potential $[8,38]$ when carbon atoms are introduced into the layered structure instead of boron atoms responsible for superconductivity. Taking into account these two mechanisms, we obtain the dependence which adequately describes the experimental data $\left(\mathrm{MgB}_{2-x} \mathrm{C}_{x}\right.$ and $\left.\mathrm{Mg}_{0.95} \mathrm{Cu}_{0.05} \mathrm{~B}_{2-x} \mathrm{C}_{x}\right)$. The doping with holes $(\delta<$ 0) $\left(\mathrm{Mg}_{1-x} \mathrm{Li}_{x} \mathrm{~B}_{2}\right.$ and $\left.\mathrm{Mg}_{1-x} \mathrm{Cu}_{x} \mathrm{~B}_{2}\right)$ does not change the $T_{\mathrm{c}}$ value, because the impurity is not introduced into the layer responsible for superconductivity and the atoms of lithium and copper introduced instead of magnesium atoms correspond only to the variation of the effective valence of boron. 
The decrease of the $T_{\mathrm{c}}$ value in $\mathrm{Mg}_{0.8} \mathrm{Li}_{0.2} \mathrm{~B}_{2-x} \mathrm{C}_{x}$ is due to the scattering of electrons on the impurity potential of carbon atoms. The jump of electron heat capacity $\left(C_{\mathrm{S}}-C_{\mathrm{N}}\right) / C_{\mathrm{N}}$ at $T=T_{\mathrm{c}}$ on value of $\delta$ is obtained; this jump at $\delta=0$ (which corresponds to the system of $\mathrm{MgB}_{2}$ without impurity) is equal to 0.8 . This small value is due to the overlapping of the energy bands on the Fermi surface. As this overlapping decreases (to the right or to the left from this point), the value of this jump increases and becomes equal to 1.43 (at $\delta \approx 0.06$, for example) which corresponds to the one-band system. These estimations have been made taking into account the effect of occupation of energy bands only, without consideration of the impurity scattering. The obtained results correctly reflect the transition from the two-band system to the one-band system and are in qualitative agreement with the experimental data (for details see $[86,87]$ ). The above theoretical results adequately describe the dependences which were observed during the experiment $[88,89]$.

\section{b) The upper critical fields $H_{c 2}^{(a b)}$ and $H_{c 2}^{(c)}$ in the intermetallic compound $\mathrm{MgB}_{2}$}

The experimental investigations of the $\mathrm{MgB}_{2}$ magnetic properties show a clear appearance of anisotropy of the upper critical field $H_{c 2}$ [90]. The upper critical field $H_{c 2}^{(a b)}$, which corresponds to the critical magnetic field in the plane $(a b)$, exceeds by several times the $H_{c 2}^{(c)}$ value with the $c$ axis magnetic field.

We set a problem to develop the microscopic theory of the upper critical field $H_{c 2}$ in a pure anisotropic two-band superconductor, applicable in the entire temperature interval $0<T<T_{\mathrm{c}}$, and to describe the pattern of the $H_{c 2}$ value behavior as a function of temperature in $\mathrm{MgB}_{2}$, as well as to determine the curvature of the upper critical field $H_{c 2}^{(a b)}$ and $H_{c 2}^{(c)}$ close to the temperature of superconducting transition and then to reveal the anisotropy of temperature dependence of the coefficient $\gamma_{H}=H_{c 2}^{(a b)} / H_{c 2}^{c}$. Also, we determine the effect of the mechanism of energy bands occupation on the values $T_{\mathrm{c}}$ and $H_{c 2}^{a b}$ when the system is doped with electrons or holes. Investigations of two-band systems are based on the microscopic approximation of the theory of superconductivity [91-93].

Herewith, the following peculiarities of the $\mathrm{MgB}_{2}$ band structure are taken into account: mutual arrangement of the energy bands, the presence of overlapping of the two-dimensional $\sigma$-band and the three-dimensional $\pi$-band, differences of the topologies of Fermi surface cavities of the considered bands.

The values of the magnetic fields given above are determined based on the Ginsburg-Landau equations for a two-band system. Thus, we apply the technique of Maki and Tsuzuki, extended for the two-band case [92] with the accounting of $\mathrm{MgB}_{2}$ compound band structure peculiarities (for details see [94-96]). This technique permits to obtain the analytic solutions for the critical fields $H_{c 2}^{(a b)}$ and $H_{c 2}^{(c)}$ in the low temperatures range $\left(T \ll T_{\mathrm{c}}\right)$ and near the critical temperature $\left(T_{\mathrm{c}}-T \ll T_{\mathrm{c}}\right)$. The allowance for anisotropy results in the anomaly of physical characteristics of the $\mathrm{MgB}_{2}$ compound. The proposed technique permits to simultaneously consider both pure anisotropic two-band superconductor and intermetallic compound $\mathrm{MgB}_{2}$ with the $\mathrm{Mg}$ and $\mathrm{B}$ atoms replaced by other elements of the periodic table. Let us present the results and calculations for the upper critical field $H_{c 2}^{(a b)}$ and $H_{c 2}^{(c)}$ which were obtained based on the constructed two-band theory. We use the constants of the electron-phonon interaction $\lambda_{n m}$ and $\mu_{0}$ (see section 5) which correspond to $\mathrm{MgB}_{2}$ as well as $\lambda=\frac{v_{1}}{v_{2}}=0.8$ (the relation of electron velocities on the difference cavities of the Fermi surface) [86]. The parameter $\varepsilon$ defined by the declination of $\sigma$-band from two-dimensionality is taken as $\varepsilon=0.31$. This value gives the closest approach to the experimental results.

We obtain $H_{c 2}^{(a b)} \gg H_{c 2}^{(c)}$. This result is in a good agreement both with the results of theoretical works and with the experimental data. The strong anisotropy of the upper critical field is explained by a weak dispersion of electron energy in the $z$ axis direction and by the low value of the average electron velocity on the Fermi surface in this direction. In case of replacing $\mathrm{Mg}$ and B by other chemical elements, which furthers the doping with electrons (the chemical potential increasing), the upper critical field as a function of temperature behavior is similar to the pure $\mathrm{MgB}_{2}$ case. However, the values of these quantities decrease in comparison with the case of pure $\mathrm{MgB}_{2}$. There takes place the correlation between the temperature of superconductive transition and the values of upper critical field with the chemical potential increasing. The hole concentration increasing has no 
effect on the superconductive temperature and the upper critical field values. The dependence [96] of critical temperatures of the doped and pure $\mathrm{MgB}_{2}$ as well as the dependence of the upper critical fields on electronic density (the chemical potential $\mu$ ) are given. We see that all the quantities decrease with increasing electronic density of the charge carriers at $\mu>0.74 \mathrm{eV}$ and remain constant at $\mu<0.74 \mathrm{eV}$. Consequently, the hole doping leaves the values of the temperature of superconducting transition and the upper critical field constant. An essential dependence of the anisotropy coefficient $\gamma_{H}$ on temperature in the pure $\mathrm{MgB}_{2}\left(\mu_{0}=0.74 \mathrm{eV}\right)$ and doped $\mathrm{MgB}_{2}(\mu=0.76 \mathrm{eV})$ was obtained. The results are in good agreement with the experimental data on the magnetic properties both of pure intermetallic compound $\mathrm{MgB}_{2}$ and the one doped with electrons and holes (see [97], for example). This counts in favor of the ability of the two-band model considered above to describe the real material properties and the ability to explain the anomalies of physical properties caused by the anisotropy of the system. Let us note that the effect of energetic band filling was considered as the main mechanism of the effect of a substitutional impurity. It was assumed that the scattering on the impurity potential is weak. The allowance for the electron scattering on impurity essentially complicates the results for the systems where the impurity scattering is strong.

\section{Conclusions}

This work is put forward for publication with the purpose of turning the scientists' mind to the information which is related to the two-band superconductor properties and which was obtained by the Moldavian physicists with professor V.A. Moskalenko at the head, who is the creator of the multiband superconductivity model. Thus, we underline the essential contribution of the N.N. Bogolyubov's school to the development of superconductivity in this direction. Let us note that our theory contains the classical results which are related to the essential distinction of the two-band superconductor properties from the one-band ones not only in a quantitative sense but also in a qualitative sense. These approaches and results had been obtained long before the discovery of HTSC and superconductivity in $\mathrm{MgB}_{2}$. The theory adequately describes all kinds of anomalies of physical characteristics of multiband superconductors (for example, $\mathrm{MgB}_{2}$ ). The great number of noncuprated compounds are obtained nowadays, such as $\left.\mathrm{M}_{\left[\mathrm{O}_{1-x}\right.} \mathrm{F}_{x}\right] \mathrm{FeAs}(\mathrm{M}=\mathrm{La}$, $\mathrm{Pr}, \mathrm{Ce}$ ) and others, for which, in particular, the presence of overlapping on the Fermi surface is essential. Consequently, the works on the two-band theory of superconductivity can be the base, to some extent, for the investigation of these new compound properties. We adduced not nearly all the investigations on the multiband superconductors theory in this work, because it is impossible to include all the authors. We should like to extend thanks to all the Moldavian physicists, who showed interest in this problem and who made contribution to its solution according to their possibilities.

\section{References}

1. Bogolyubov N.N., Zubarev D.N., Tserkovnikov Yu.A., Dokl. Akad. Nauk SSSR, 1957, 117, 788.

2. Bogolyubov N.N., Zh. Exp. Teor. Fiz., 1958, 34, 58; ibid., 73.

3. Bogolyubov N.N., Tolmacev V.V., Shirkov D.V. The New Method in Theory of Supraconductivity. Nauka, Moskow, 1958.

4. Bogolyubov N.N., Usp. Fiz. Nauk, 1959, 67, 549.

5. Moskalenko V.A., Fiz. Met. Metalloved., 1959, 8, 503;Phys. Met. Metalog., 1959, 8, 25.

6. Suhl H., Matthias B.T., Walker L.R., Phys. Rev. Lett., 1959, 3, 552.

7. Moskalenko V.A., Palistrant M.E., Dokl. Akad. Nauk SSSR, 1965, 162, 539 [Sov. Phys. Dokl., 1965, 10, 457].

8. Moskalenko V.A., Palistrant M.E., Zh. Exp. Teor. Fiz., 1965, 49, 770 [Sov. Phys. JETP, 1965, 22, 536].

9. Moskalenko V.A., Kon L.Z., Palistrant M.E. - In: Proc. of Low Temperature Conference LT-X, Moscow, 1967, p. 181.

10. Moskalenko V.A., Kon L.Z., Zh. Exp. Teor. Fiz., 1966, 50, 724.

11. Moskalenko V.A., Fiz. Met. Metalloved., 1967, 23, 585.

12. Moskalenko V.A., Palistrant M.E., Statistical Physics and Quantum Field Theory. Nauka, Moskow, p. 262, 1973 (in Russian). 
13. Moskalenko V.A., Kon L.Z., Palistrant M.E., Low-Temperature Properties of Metals with BandSpectrum Singularities. Shtiintsa, Kishinev, 1989 (in Russian);

http://www.theory.nipne.ro/ṽbarsan/ebooks/Mosk_2008.pdf (in English).

14. Moskalenko V.A., Zh. Exp. Teor. Fiz., 1966, 51, 1163 [Sov. Phys. JETP, 1966, 24, 780].

15. Moskalenko V.A. Electromagnetic and Kinetic Properties of Superconducting Alloys with Overlapping Energy Bands. Stiintsa, Kishinev, 1976, (in Russian).

16. Moskalenko V.A. Methods of Studying Electronic Densities of States in Superconducting Alloys. Stiintsa, Kishinev, 1974 (in Russian).

17. Palistrant M.E. Preprint arXiv: cond-mat/0305356, 2003.

18. Kon L.Z. Preprint arXiv: cond-mat/0309707, 2003.

19. Tilley R., Proc. Phys. Soc., 1964, 84, 573; ibid., 1965, 86, 289.

20. Geilikman B.T., Kresin V.Z., Usp. Fiz. Nauk, 1969, 99, 51.

21. Geilikman B.T., Usp. Fiz. Nauk, 1966, 88, 327.

22. Loktev V.M., Quick R.M., Sharapov S.G., Phys. Rep., 2001, 349, 1-123.

23. Baranov M.A., Kagan Yu.M., Zh. Exp. Teor. Fiz., 1992, 102, 313 [Sov. Phys. JETP, 1992, 75, 165].

24. Alekseevskiyi N.E., Homskiyi D.I., Usp. Fiz. Nauk, 1985, 147, 767.

25. Bednorz J.G., Muller K.A., Z. Phys., 1986, 64, 189.

26. Krakauer H., Pickett E., Z. Phys., 1988, 60, 1665.

27. J. F. Herman, R. V. Kasowski, W. G. Hsu, Phys. Rev. B. 36, 6904 (1987).

28. Gorbar E.V., Loktev V.M., Sharapov S.G., Fiz. Nizk. Temp., 1995, 21, 21.

29. Helm M., Forsthofer F., Keller J., Phys. Rev. B, 1996, 53, 14481.

30. Kochorbe F.G., Palistrant M.E., Zh. Exp. Teor. Fiz., 1998, 114, 1047; J. Exp. Theor. Phys., 1998, 87, 570 .

31. Verkin B.I., Bondurian B.B., Baril'nik A.S., Fiz. Nizk. Temp., 1987, 13, 771.

32. Moskalenko V.A., Golub A.A., Driuma V.C., Fiz. Met. Metalloved., 1969, 27, 415.

33. Palistrant M.E., Kochorbe F.G., Fiz. Nizk. Temp., 2000, 26, 1077;Low Temp. Phys., 2000, 26, 799.

34. Lee D.H., Ihm J., Solid. State Commun., 1987, 62, 81.

35. Moskalenko V.A., Palistrant M.E., Vakalyuk V.M., Mechanisms of High-Temperature Superconductivity. Joint Inst. for Nucl. Research, Dubna, 1988, p. 34 (in Russian).

36. Moskalenko V.A., Palistrant M.E., Vakalyuk V.M., Fiz. Nizk. Temp., 1989, 15, 378.

37. Galaiko V.P., Bezuglyi E.V., Shumeiko V.S., Fiz. Nizk. Temp., 1987, 13, 1301.

38. Palstrant M.E., Kochorba F.G., Fiz. Nizk. Temp., 2000, 26, 1077;Low Temp. Phys., 2000, 26, 799.

39. Moskalenko V.A., Palistrant M.E., Vakalyuk V.M., Padure I.V., Solid State. Commun., 1989, 69, 747.

40. Moskalenko V.A., Palistrant M.E., Vakalyuk V.M. - In Proc. of 10th Int. Symp. on the Jahn-Teller Effect, Kishinev, 1989, p. 88.

41. Moskalenko V.A., Palistrant M.E., Vakalyuk V.M. - In Proc. of Eighth Int.Conf. on Ternary and Multinary Compounds, Kishinev, 1990, p. 57.

42. Palistrant M.E., Kochorbe F.G., Physica C, 1992, 194, 351.

43. Palistrant M.E., Vakalyuk V.M., Sverkhprovodimost': Fiz. Khim. Tekh., 1990, 3, 557.

44. Palistrant M.E., Kochorbe F.G., Quantum-Field Methods of Investigating High - Temperature Superconductors and Disordered Systems. Shtiintsa, Kishinev, 1992 (in Russian).

45. Kalalb M.G., Kochorbe F.G., Palistrant M.E., Teor. Mat. Fiz., 1992, 91, 483.

46. Palistrant M.E., Kalalb M.G., Izv. Akad. Nauk, Resp. Moldova, 1992, 1(7), 70.

47. Moskalenko V.A., Palistrant M.E., Vakalyuk V.M., Usp. Fiz. Nauk, 1991, 161, 155 [Sov. Phys. Usp., 1991, 34, 717;Preprint arXiv: cond-mat/03099671].

48. Kresin V., Wolf S.A., Phys. Rev. B, 1990, 41, 4278;Physica C, 1990, 169, 476.

49. Hirch J.E., Marsiglio F., Phys. Rev. B, 1991, 43, 424.

50. Binning G., Baratoff A., Hoening H.E. et al., Phys. Rev. Lett., 1980, 45, 1352.

51. Rare Earth Transition Metal Borocarbides (Nitrides) - In: Superconducting, Magnetic and Normal State Properties, eds. K.-H. Muller, V. Narozhnyi, NATO Sciences Series. II Mathematics, Physics and Chemistry, vol. 14.

52. Nagamatsu J., Nakagawa N., Muranaka T., Zenitani Y., Akimutsu J., Nature (London), 2001, 410, 63.

53. Bouquet F., Wang Y., Sheikin I. et al., Physica C, 2003, 385, 192.

54. Confield P.C., Bud'ko S.L., Finemore D.K., Physica C, 2003, 385, 1.

55. Bud'ko S.L., Lapertot G., Petrovic C. et al., Phys. Rev. Lett., 2001, 86, 1877.

56. Hinks D.G., Claus H., Jorgensen J.D., Nature, 2001, 411, 457.

57. Kotegawa H., Ishida K., Kitaoka Y., Muranaka T., Akimitsu J., Phys. Rev. Lett., 2001, 87, 127001.

58. Osborn R., Goremychkin E.A., Kolesnikov A.I. et al., Phys. Rev. Lett., 2001, 87, 017005. 
59. Wang Y., Plockowski T., Junod A., Physica C, 2001, 355, 179.

60. Pokrovskii V.L., Zh. Exp. Teor. Fiz., 1961, 40, 641 [Sov. Phys. JETP, 1961, 13, 447].

61. Pokrovskii V.L., Ryvkin M.S., Zh. Exp. Teor. Fiz., 1962, 43, 92 [Sov. Phys. JETP, 1963, 16, 67].

62. Mishonov T., Penev E. Preprint arxiv: cond-mat/0206118, 2002;Int. J. Mod. Phys. B, 2002, 16, 3573.

63. Mishonov T., Drechsler S.L., Penev E. Preprint arxiv: cond-mat/0209192 V 1, 2002;Mod. Phys. Lett., 2003, 17, 755 .

64. Mishonov T., Penev E., Indekeu J.O., Pokrovsky V.I. Preprint arxiv: cond-mat/0209342, 2003);Phys. Rev. B, 2003, 68, 104517.

65. Miranovic P., Machida K., Kogan V.G., J. Phys. Soc. Jpn., 2003, 72, 221.

66. Van der Marel V., Physica C, 1990, 165, 35.

67. Kochorbe F.G., Palistrant M.E., Zh. Exp. Teor. Fiz., 1993, 104, 3084 [JETP, 1993, 77, 442];Teor. Mat. Fiz., 1993, 96, 459 [Theoret. Mathemat. Phys., 1993, 96, 1083].

68. Schafroth M.R., Phys. Rev., 1958, 111, 70.

69. Randeria M., Duan J.M., Shich L.I., Phys. Rev. Lett., 1989, 62, 981;Phys. Rev. B, 1990, 41, 372.

70. Leggett A.J., Modern Trends in the Theory of Condensed Matter, 1980, 115, 13.

71. Gorbatsevich A.A., Tokatly I.V., Zh. Exp. Teor. Fiz., 1993, 76, 347.

72. Gorbar E.V., Gusynin V.P., Loktev V.M., Sverkhprovodimost': Fiz. Khim. Tekh., 1993, 6, 483;Fiz. Nizk. Temp., 1993, 19, 1171.

73. Loktev V.M., Quick R.M., Sharapov S.G., Phys. Rep., 2001, 349, 1-123.

74. Leggett A.J., Prog. Theor. Phys., 1966, 36, 901.

75. Palistrant M.E., Int. J. Mod. Phys. B, 2005, 19, 929-970.

76. Palistrant M.E., Teor. Mat. Fiz., 1993, 95, 104.

77. Palistrant M.E., Teor. Mat. Fiz. 105, 491 (1995).

78. Palistrant M.E., Teor. Mat. Fiz. 109, 137 (1996).

79. Palistrant M.E., J. Supercond. 10, 19 (1997).

80. Palistrant M.E., Ursu V.A., arXiv cond-mat/0602551, Apr.2006, Theor. Mat. Fiz. 149, 1393 (2006).

81. Palistrant M.E., Vakalyuk V.M., Calalb M.Gh., Phisica C, 208, 170, (1993).

82. Palistrant M.E., Teor. Mat. Fiz. 103312 (1995); Theor. Math. Phys. 103, 583 (1995).

83. Kochorbe F.G., Palistrant M.E., Physica C 298, 217 (1998).

84. Kochorbe F.G., Palistrant M.E., Zh. Exp. Teor. Fiz. 114, 1047 (1998); [JETP 87, 570 (1998)].

85. Calalb M.Gh., Palistrant M.E., Teor. Mat. Fiz. 110, 162 (1997).

86. Palistrant M.E., Ursu V.A., Exp.Teor.Fiz. 131, 59 (2007); [JETF 104,51 (2007)].

87. Palistrant M.E., Ursu V.A., J.Supercond. Nov. Magn. DOI, 10/s 10948-008-0312-5 .

88. Hofanova Z., Szabo P., Samuely P. et al., Phys.Rev.B, 70, 064520 (2004).

89. Balaselvi S.J., Bharathi A., Sastry V.S., Reddy G.L.N., Hariharan Y. Preprint arXiv: condmat/0303022, 2003.

90. Angst M., Puzniak R. - In: Focus Superconductivity, ed. B.P. Martins. Nova Sciences, 1, 2004, 1;Preprint arXiv: cond-mat/0304503, 2003.

91. Gor'kov L.P., Zh. Exp. Teor. Fiz., 1959, 37, 1918.

92. Maki K., Tsuzuki T., Phys. Rev., 1965, 139, 868.

93. Moskalenko V.A., Zh. Exp. Teor. Fiz., 1966, 51, 1163.

94. Moskalenko V.A., Palistrant M.E., Ursu V.A., Teor. Mat. Fiz., 2008, 154, 113 [Theor. Math. Phys., 2008, 154, 94].

95. Palistrant M.E., Cibotari I.D., Ursu V.A., Moldavian J. Phys. Sci., 2008, 7(3), 292.

96. Palistrant M.E., Cibotari I.D., Ursu V.A., Zh. Exp. Teor. Fiz., 2009, 136, 272 [JETP, 2009, 109, 197].

97. Angst M., Bd'ko S.Z., Wilke R.H. et al., Phys. Rev. B, 2005, 71, 144512. 


\title{
Теорія термодинамічних і магнітних властивостей багатозонних надпровідників
}

\author{
М.€. Палістрант \\ Інститут прикладної фізики, Кишинів, Молдова
}

Отримано 10 вересня 2009 р., в остаточному вигляді - 2 листопада 2009 р.

Формулюються основні стани розвитку теорії надпровідних систем із енергетичними зонами, що перекриваються. Приводяться основні поклики на класичні роботи авторів цієї теорії (проф. В.А. Москаленко та його співробітники) доповнений іншими роботами з високотемпературної надпровідності. Описано певні особливості двозонної моделі, що приводять до цілком нових результатів у порівнянні з однозонною моделлю. Обговорюються можливості використання двозонної моделі до опису термодинамічних і магнітних властивостей сполуки MgB2.

Ключові слова: багатозонна надпровідність, термодинамічні властивості, низькі густини носіїв, верхнє критичне поле, $\mathrm{MgB}_{2}$

PACS: $74.25 . \mathrm{Ha}, 74.20 . \mathrm{Fg}, 74.70 . \mathrm{A}$ 
